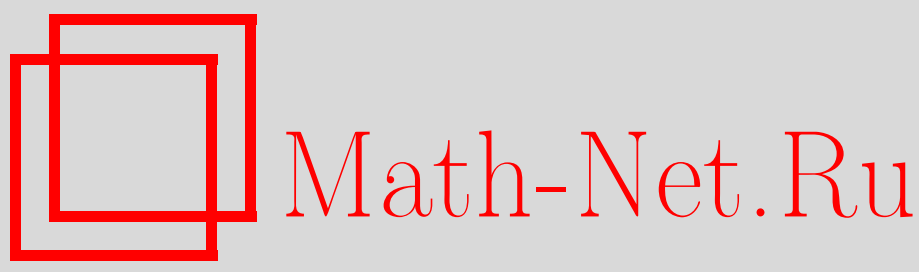

В. В. Козлов, В. В. Тен, Топология областей возможности движения интегрируемых систем, Матем. сб., 1996, том 187, номер 5, 59-64

DOI: https://doi.org/10.4213/sm127

Использование Общероссийского математического портала Math-Net.Ru подразумевает, что вы прочитали и согласны с пользовательским соглашением

http://www . mathnet.ru/rus/agreement

Параметры загрузки:

IP: 52.23 .180 .231

26 апреля 2023 г., $13: 16: 26$ 
УДК $517.9+531.01$

\author{
В. В. Козлов, В.В. Тен
}

\title{
Топология областей возможности движения интегрируемых систем
}

\begin{abstract}
Рассматриваются аналитические обратимые системы с двумя степенями свободы на фиксированном трехмерном многообразии уровня интеграла энергии. Предполагается, что это многообразие компактно и не имеет особых точек (равновесий исходной системы). Естественная проекция энергетического многообразия на двумерное конфигурационное пространство называется областью возможности движения. В ориентируемом случае это сфера с $k$ дырами и $p$ приклеенными ручками. Известно, что если $k=0$ и $p \geqslant 2$, то система не имеет непостоянного аналитического интеграла на соответствующем уровне интеграла энергии. Оказывается, для областей возможности движения с краем ситуация совсем иная. Основной резултат состоит в следующем: имеются примеры аналитически интегрируемых систем, для которых числа $p$ и $k \geqslant 1$ произвольны.

Библиографйл: 10 названий.
\end{abstract}

\section{§1. Введение. Основной результат}

Рассмотрим обратимую динамическую систему с двумя степенями свободы. Пусть $M$ - ее конфигурационное пространство; это двумерное многообразие. $\Phi$ азовое пространство четырехмерно; оно совпадает с пространством кокасательного расслоения $T^{*} M$.

Функция Гамильтона $H: T^{*} M \rightarrow \mathbb{R}$ обратимой системы имеет вид

$$
H=T+V \text {, }
$$

где

$$
T=\frac{1}{2} \sum g_{i j}(x) y_{i} y_{j}
$$

- кинетическая энергия (риманова метрика на $M$ ) - положительно определенная квадратичная форма по импульсам $y_{1}, y_{2}$, а $V: M \rightarrow \mathbb{R}$ - потенциальная энергия (функция от координат $x_{1}, x_{2}$ на $M$ ). В дальнейшем предполагается, что $M$ имеет структуру ориентированного аналитического многообразия, а $H$-аналитическая функция на $T^{*} M$. В случае неориентируемого $M$ имеется естественное двулистное накрытие $N \rightarrow M$, где $N$ уже ориентируемо, причем динамическая система на $T^{*} M$ "поднимается" до обратимой системы на $T^{*} N$.

Работа выполнена при финансовой поддержке Российского фонда фундаментальных исследований (грант № 93-013-16244) и Международного научного фонда (грант № МСУ 000). 
Уравнения Гамильтона

$$
\dot{x}_{k}=\frac{\partial H}{\partial y_{k}}, \quad \dot{y}_{k}=-\frac{\partial H}{\partial x_{k}} \quad(k=1,2)
$$

имеют интеграл энергии (1.1). Зафиксируем его постоянную, которую обозначим $h$. Трехмерный уровень интеграла энергии

$$
\Sigma_{h}=\{x, y: H=h\}
$$

будет регулярным аналитическим многообразием, если на $\Sigma_{h}$ нет критических точек функции $H$, т.е. состояний равновесия рассматриваемой системы. Это предположение, очевидно, эквивалентно условию, что $h$ не является критическим значением потенциальной энергии $V$. В дальнейшем рассматривается случай, когда $\Sigma$ компактно.

Естественная проекция $T^{*} M \rightarrow M$ (точка $(x, y)$ переходит в точку $\left.x\right)$ переводит $\Sigma_{h}$ в некоторую компактную область (возможно с краем) $B_{h}$ на $M$. Эта область называется областью возможсности движсения. Так как $T \geqslant 0$, то

$$
B_{h}=\{x \in M: V(x) \leqslant h\} .
$$

Геометрия и динамика областей возможности движения обсуждается в [1].

Предположим теперь, что система (1.2) на $\Sigma$ допускает непостоянньй аналитический интеграл

$$
F: \Sigma \rightarrow \mathbb{R}
$$

По теореме Лиувилля некритические поверхности уровня функции $F$ будут объединением двумерных торов с условно-периодическими траекториями. Так как аналитическая функция на компактном многообразии имеет конечное число критических значений, то $\Sigma$ разбивается на конечное число кусков, "регулярно" расслоенных на двумерные торы.

Оказывается, свойство полной интегрируемости системы (1.2) на $\Sigma$ существенно зависит от топологии области возможности движения $B$. Первый результат в этом направлении получен в работе [2]: если $\partial B=\varnothing(M$ компактно и $h>\max V)$ и род $p$ поверхности $B=M$ (число ручек) $\geqslant 2$, то динамическая система на $\Sigma$ не имеет непостоянного аналитического интеграла. С другой стороны, известны многочисленные примеры интегрируемых задач, когда $p=0$ (сфера) и $p=1$ (тор). Впоследствии были получены новые доказательства этого результата, основанные на других идеях (см. [3]-[6]).

Рассмотрим теперь случай, когда граница $\partial B$ не пуста. Топологическая классификация таких областей хорошо известна. Это сфера с $k \geqslant 1$ дырами и $p \geqslant 0$ ручками. Число $p$ называется родом области $B$. Наш основной результат составляет

Теорема 1. Для любых $k \geqslant 1$ и $p \geqslant 2$ существуют аналитическая система (1.2) и значение әнергии $h$ такие, что

1) ограничение системы (1.2) на $\Sigma_{h}$ допускает непостоянный аналитический интеграл,

2) компактная область $B_{h}$ гомеоморфна сфере с $k$ дьрами и р ручками. 
Таким образом, область возможности движения с краем аналитически интегрируемой системы с двумя степенями свободы может быть устроена топологически сколь угодно сложно.

В связи с этим замечанием полезно сравнить теорему 1 с результатом работы [7]. Пусть $V \equiv 0$, и найдется двумерное многообразие с краем $M_{0} \subset M$ такое, что $\chi\left(M_{0}\right)<0$, и граница $M_{0}$ геодезически выпукла. Тогда уравнения (1.2) не имеют дополнительного аналитического интеграла. Например, если $M_{0}-$ диск с $k \geqslant 2$ дырами, то $\chi\left(M_{0}\right)=1-k<0$.

\section{§2. Случай $p \leqslant 1$}

Наиболее просто теорема 1 доказывается в случаях, когда $p=0$ и $p=1$. Предъявим системы, аналитически интегрируемые на $\Sigma$, причем проекции $\Sigma$ на $M$ (область возможности движения) имеет любое заданное число дыр.

Положим сначала $p=0$. Пусть $M=\mathbb{R}^{2}=\left\{x_{1}, x_{2}\right\}$, кинетическая энергия задает плоскую метрику

$$
T=\left(y_{1}^{2}+y_{2}^{2}\right) / 2,
$$

потенциал $V$ равен сумме

$$
\begin{gathered}
f\left(x_{1}\right)+g\left(x_{2}\right), \\
f(z)=T_{4}(z)=8 z^{4}-8 z^{2}+1, \quad g(z)=T_{2 k+2}(z) .
\end{gathered}
$$

Здесь $T_{n}(z)=\cos (n \arccos z)$ - многочлењы Чебышёва.

Ясно, что $V\left(x_{1}, x_{2}\right) \rightarrow+\infty$, когда $x_{1}^{2}+x_{2}^{2} \rightarrow \infty$. Потенциальная энергия имеет ровно $k$ локальных максимумов, где эта функция принимает одно и то же значение, равное 2. Положим $h=2-\varepsilon$, где $\varepsilon$ - малое положительное число. Легко понять, что тогда $B_{h}$ гомеоморфно диску с $k$ дырами.

Остается заметить, что обратимая система с кинетической энергией (2.1) и потенциалом (2.2) вполне интегрируема. Она допускает дополнительный аналитический интеграл

$$
F=\frac{y_{1}^{2}}{2}+f\left(x_{1}\right)
$$

независимьй от интеграла энергии $H=T+V$.

Для $p=1$ и произвольного $k \geqslant 0$ также можно указать обратимую систему, интегрируемую разделением переменных. Пусть

$$
\begin{gathered}
M=\mathbf{T}^{2}=\left\{x_{1}, x_{2} \bmod 2 \pi\right\}, \\
T=\frac{y_{1}^{2}+y_{2}^{2}}{2}, \quad V=\cos x_{1}+\cos (k+1) x_{2} .
\end{gathered}
$$

Легко проверить, что при $h=2-\varepsilon, \varepsilon>0$-мало, область $B_{h}$ гомеоморфна диску с одной ручкой и $k$ дырами. Дополнительным аналитическим интегралом служит, например, функция

$$
F=\frac{y_{1}^{2}}{2}+\cos x_{1}
$$




\section{§3. Случай $p>1$}

Построение аналитически интегрируемой системы с областью возможности движения рода $p>1$ основано на идее, изложенной в $§ 2$.

Рассмотрим на евклидовой плоскости $\mathbb{R}^{2}=\left\{x_{1}, x_{2}\right\}$ квадрат

$$
\Pi=[-\pi, \pi] \times[-\pi, \pi]
$$

и зададим кинетическую и потенциальную энергии формулами

$$
T=\frac{y_{1}^{2}+y_{2}^{2}}{2}, \quad V=-\cos x_{1}-\cos x_{2} ; \quad y_{i}=\dot{x}_{i} \text {. }
$$

При $h=1$ область возможности движения $B_{h} \subset$ П - это криволинейный крест, пересекающийся с границей $\partial П$ по отрезкам

$$
\begin{aligned}
a_{1} & =\{-\pi\} \times\left[-\frac{\pi}{2}, \frac{\pi}{2}\right], & a_{2} & =\{\pi\} \times\left[-\frac{\pi}{2}, \frac{\pi}{2}\right], \\
b_{1} & =\left[-\frac{\pi}{2}, \frac{\pi}{2}\right] \times\{-\pi\}, & b_{2} & =\left[-\frac{\pi}{2}, \frac{\pi}{2}\right] \times\{\pi\} .
\end{aligned}
$$

Пусть

$$
K=\left\{\left(x_{1}, x_{2}\right) \in \Pi: V\left(x_{1}, x_{2}\right) \leqslant \frac{3}{2}\right\}
$$

- бо́льший крест с граничными отрезками $\alpha_{1} \supset a_{1}, \alpha_{2} \supset a_{2}, \beta_{1} \supset b_{1}$ и $\beta_{2} \supset b_{2}$.

Рассмотрим теперь $2 p+k-2$ экземпляров криволинейного креста (3.2). Декартовы координаты в $i$-м кресте $K_{i}$ обозначим $x_{1}^{i}$ и $x_{2}^{i}$. Кинетическую и потенциальную энергии в $K_{i}$ зададим формулами (3.1), в которых переменные $x_{k}, y_{k}$ заменены на $x_{k}^{i}, y_{k}^{i}$. Граничные отрезки креста $K_{i}$ обозначим $\alpha_{1}^{i}, \alpha_{2}^{i}, \beta_{1}^{i}, \beta_{2}^{i}$. Два креста $K_{i}$ и $K_{j}$ можно склеить, например, по отрезкам $\alpha_{1}^{i}$ и $\alpha_{2}^{j}$, отождествив точки с координатами $\left(-\pi, x_{2}^{i}\right)$ и $\left(\pi, x_{2}^{j}\right)$. Такую склейку будем обозначать символом $\left(\alpha_{1}^{i}, \alpha_{2}^{j}\right)$. Это склеивание можно осуществить таким образом, чтобы в результате получилось аналитическое двумерное многообразие (с краем). Аналогично определяется операция склеивания $\left(\beta^{i}, \beta^{j}\right)$. Склеим теперь $K_{1}, \ldots, K_{2 p+k-2}$ по формуле

$$
\begin{aligned}
& \left(\beta_{1}^{1}, \beta_{2}^{1}\right)+\left(\alpha_{1}^{1}, \alpha_{1}^{2}\right)+\left(\alpha_{2}^{1}, \alpha_{2}^{2}\right)+\cdots+\left(\beta_{1}^{2 p-2}, \beta_{1}^{2 p-1}\right)+\left(\beta_{2}^{2 p-2}, \beta_{2}^{2 p-1}\right) \\
& \quad+\sum_{i=2}^{k}\left(\left(\alpha_{1}^{2 p+i-3}, \alpha_{2}^{2 p+i-2}\right)+\left(\beta_{1}^{2 p+i-2}, \beta_{2}^{2 p+i-2}\right)\right)+\left(\alpha_{1}^{2 p+k-2}, \alpha_{2}^{2 p-1}\right)
\end{aligned}
$$

Символ “+” означает последовательное повторение склеивания. В результате получим аналитическое компактное многообразие $M$ с краем.

Лемма. Многообразие $M$ гомеоморфно сфере с $k$ дырами и р приклеенными ручками.

Это утверждение будет доказано ниже.

Ввиду периодичности потенциальная энергия $V: M \rightarrow \mathbb{R}$ будет аналитической функцией. Область возможности движения

$$
B_{1}=\{V \leqslant 1\} \subset M
$$


очевидно, гомеоморфна $M$. Построенная обратимая механическая система аналитически интегрируема. Действительно, зададим в $T^{*} K_{i}(i=1, \ldots, 2 p+k-2)$ функцию $F$ формулой

$$
\frac{\left(y_{1}^{i}\right)^{2}}{2}-\cos x_{1}^{i} .
$$

Ясно, что это - интеграл построенной обратимой гамильтоновой системы. Функция $F$ определена почти всюду на $T^{*} M$ и по построению многообразия $M$ она продолжается по непрерывности до аналитической функции на всем фазовом пространстве $T^{*} M$. Теорема доказана.

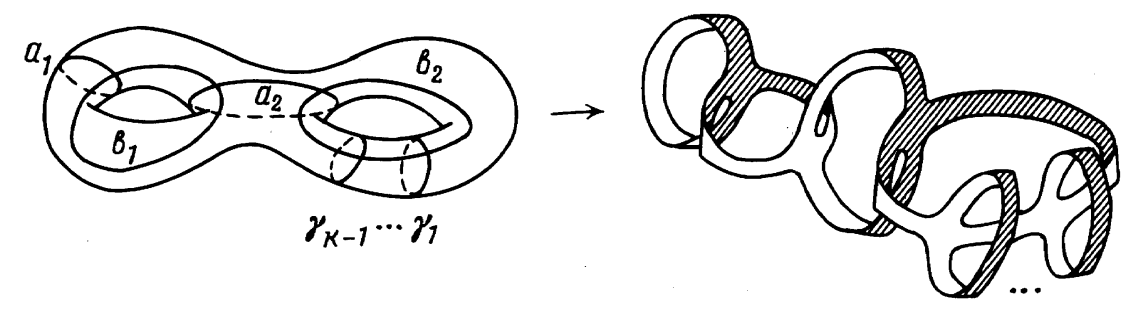

ДокАЗАТЕЛЬСТво ЛЕммы. Рассмотрим замкнутую поверхность рода $p$ (на рис. $p=2)$ и $2 p$ "канонических" замкнутых циклов $a_{1}, b_{1}, \ldots, a_{p}, b_{p}$; пусть $\gamma_{1}, \ldots, \gamma_{k-1}$ - гомологичные циклы, пересекающие $b_{p}$ (как показано на рис.). Если разрезать поверхность по этим $2 p+k-1$ циклам, то поверхность распадается на $k$ кусков, каждый из которых гомеоморфен диску. Окружим теперь указанные циклы тонкими полосками. Область на поверхности вне этих полосок будет несвязным объединением $k$ дисков. Следовательно, объединение всех полосок (см. рис.) - поверхность с краем рода $p$, граница которого имеет ровно $k \geqslant 1$ связных компонент. Нетрудно проверить, что она получается путем склеивания $2 p+k-2$ крестов по формуле (3.3). Лемма доказана.

\section{§4. Заключительные замечания}

1. Функция $F: T^{*} M \rightarrow \mathbb{R}$ называется условным (по Биркгофу) интеграломгамильтоновой системы (1.2), если $\dot{F}=0$ на некоторой поверхности $\Sigma_{h}=\{H=h\}$. Теорема 1 допускает следуюшее уточнение.

ТЕОРема 2. Для любой компактной поверхности $N$ с краем найдутся аналитическая обратимая гамильтонова система и значение полной энергии $h$ такие, что

1) $B_{h}$ гомеоморфно $N$

2) существует нетривиальный условный интеграл в виде полинома второй степени по импульсам.

Таким образом, для областей возможности движения с краем нет никаких топологических препятствий к сушествованию условных квадратичных интегралов. Интересно отметить, что для условных интегралов, линейных по импульсам, такие 
препятствия существуют. Как доказано в [9], если при $H=h$ имеется условный линейный интеграл, то эйлерова характеристика области $B_{h}$ неотрицательна.

2. Было бы интересным исследовать задачу о препятствиях к существованию условных полиномиальных интегралов для необратимых систем с гироскопическими силами, когда $\partial B \neq \varnothing$. Для случая, когда $B$ совпадает со всем $M$, эта задача исследована в [10]. Если род $M$ больше 1, то условных полиномиальных интегралов нет. Если же $M$ гомеоморфно тору, то необходимое условие интегрируемости по Биркгофу состоит в следующем: интеграл 2-формы гироскопических сил по тору равен нулю.

3. Когда $\partial B \neq \varnothing$, то вопрос о топологических препятствиях к полной интегрируемости для систем со многими степенями свободы остается пока открытьм. Здесь речь должна идти об условиях сушествования $n$ инволютивных интегралов, где $n$ - число степеней свободы системы.

\section{Список литературы}

1. Козлов В. В. Вариационное исчисление в целом и классическая механика // УМН. 1985. T. 40. № 2. C. 33-60.

2. Козлов $B$. . Т. Топологические препятствия к интегрируемости натуральных механических систем // ДАН СССР. 1979. Т. 249. №6. С. 1299-1302.

3. Колокольцов В. Н. Геодезические потоки на двумерных многообразиях с дополнительным по скорости первым интегралом // Изв. АН СССР. Сер. матем. 1982. Т. 46. № 5. С. $994-1010$.

4. Тайманов И. А. Топологическиепрепятствия к интегрируемостигеодезических потоков на неодносвязных многообразиях // Изв. АН СССР. Сер. матем. 1987. Т. 51. № 2. C. $429-435$.

5. Болотин С.В. Двоякоасимптотические траектории минималњных геодезических // Вестн. МГУ. Сер. 1. Матем., мех. 1992. № 1. С. 92-96.

6. Paternain G. P. On the topology of manifolds with completely integrable geodesic flows // Ergod. Th. and Dynam. Sys. 1992. V. 12. P. 109-121.

7. Болотин С. В. Неинтегрируемость задачи $n$ центров при $n>2$ // Вестн. МГУ. Сер. 1 . Матем., мех. 1984. № 3. С. 65-68.

8. Козлов В.В. Интегрируемость и неинтегрируемость в гамильтоновой механике // УМН. 1983. Т. 38. № 1. С. 3-67.

9. Абраров Д. Л. Топологические препятствия к существованию условно-линейных интегралов // Вестн. МГУ. Сер. 1. Матем., мех. 1984. №6. С. 72-75.

10. Болотин C. В. О первых интегралах систем с гироскопическими силами // Вестн. МГУ. Сер. 1. Матем., мех. 1984. №6. С. 75-82.

Московский государственный

Поступила в редакцию

университет им. М.В. Ломоносова

03.10 .1995 